\title{
Atmospheric Radio Noise Bursts in the LF Band at Bangalore
}

\author{
S. V. C. Aiya and K. N. Lakshminarayan \\ Indian Institute of Science, Bangalore, India
}

(Received February 9, 1965; revised April 26, 1965; May 21, 1965)

\begin{abstract}
An atmospheric radio noise burst represents the radiation received from one complete lightning flash at the frequency to which the receiver is tuned and within the receiver bandwidth. The characteristics of such noise bursts arising from sources at various distances have been investigated. The group of bursts with the highest amplitudes present at any given time can be investigated separately by adjusting the sensitivity of the receiver. The number of bursts in a group due to sources up to about $1000 \mathrm{~km}$ mostly lies between 10 and 40 per minute. The short and long term amplitude and time characteristics of these bursts have been investigated. The distributions are found to be $\log$ normal. In the case of distributions of parameters studied over a short period of time, the standard deviation is practically constant. The long term characteristics indicate that there are systematic variations from 12 to $20 \mathrm{hr}$ IST.
\end{abstract}

\section{Introduction}

This paper gives a report of the experimental results on some characteristics of atmospheric radio noise bursts as observed at 105 and $280 \mathrm{kc} / \mathrm{s}$ with a receiver bandwidth of $4 \mathrm{kc} / \mathrm{s}$ at $6 \mathrm{~dB}$ and below. The report is based on data collected at Bangalore $(12.58 \mathrm{~N}$, 77.35 E) from December 1961 to November 1962 during 12 to $24 \mathrm{hr}$ IST. (Indian Standard Time is $5 \mathrm{hr}$ $30 \mathrm{~min}$ ahead of Greenwich Mean Time). The procedure followed for the collection and assessment of data was as previously described and discussed [Aiya, 1954, 1958 and 1962; Lakshminarayan, 1962).

The subject matter of the paper is treated in the following order. Sources of noise bursts are classified. The observed features of noise bursts are described and discussed. The short term 'amplitude' and 'time' characteristics are then deduced from the data. There follows a discussion of a procedure for the study of the long term characteristics. They are then deduced from the data on the basis of the discussion. A concluding section reviews the entire work.

\section{Sources of Noise Bursts}

Atmospheric noise bursts arise from the radiation fields of electrical discharges in lightning flashes accompanying thunderstorms. The duration of a noise burst is about the same as that of the electrical discharge. In the frequency range, 0.1 to $15 \mathrm{Mc} / \mathrm{s}$, the principal sources of noise radiation are the electrical discharges occurring inside the cloud. Since such discharges are associated with all types of flashes, every flash radiates a noise burst. In the tropics, the average height of the cloud base is about $3 \mathrm{~km}$ above the ground level. Hence, noise burst radiators are generally over $3 \mathrm{~km}$ above ground level.
Noise bursts arising from sources lying within 200 to $300 \mathrm{~km}$ can be expected to be received by the direct ray. Hence, all such sources are described here as local sources. The reception of noise bursts arising from sources lying at greater distances can be accomplished by a variety of modes of propagation depending on frequency, time of day, etc. For a place like Bangalore, there is always a reasonable number of nonlocal sources lying within about $1000 \mathrm{~km}$. For such sources, the attenuation due to propagation for the noise bursts received is not always very large and these bursts appear distinctly above the background. In view of this fact, nonlocal sources lying within about $1000 \mathrm{~km}$ will be described as near sources. All sources other than local and near ones will be referred to as distant sources. Observations at Bangalore have shown that noise bursts arising from local and near sources at 105 and $280 \mathrm{kc} / \mathrm{s}$ are, most of the time, distinct and well separated.

\section{Noise Bursts}

\subsection{Nature of Noise Bursts}

The audio frequency output arising from atmospheric noise bursts at the detector of a superheterodyne receiver with no automatic gain control has been fed quite frequently to a high sensitivity and high fidelity level recorder, and records have been obtained. The tracing of a typical record is reproduced in figure 1. It was taken at a time when noise bursts arising from both local and near sources were present. The paper speed was $10 \mathrm{~mm} / \mathrm{sec}$ and the writing speed was $700 \mathrm{~mm} / \mathrm{sec}$. Portions like those marked $\mathrm{X}$ correspond to periods when there were neither local nor near sources. Ordinarily, such portions are 


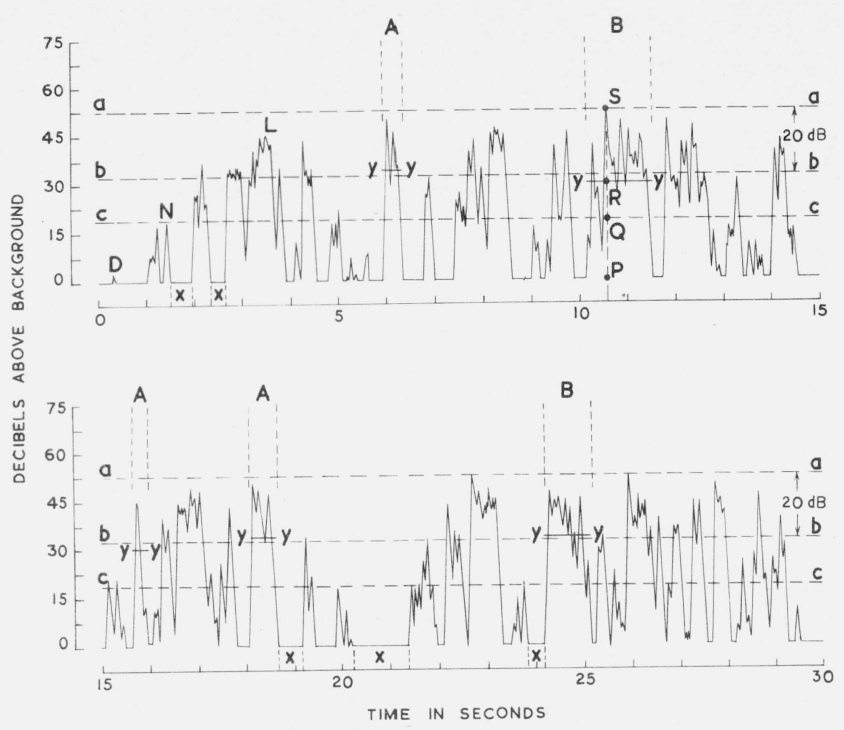

FIGURE 1. Typical record of detected audio frequency voltage arising out of noise bursts at $105 \mathrm{kc} / \mathrm{s}$. (Receiver bandwidth $-4 \mathrm{kc} / \mathrm{s}$ at $6 \mathrm{~dB}$ down.)

more numerous than in figure 1 . The line cc, represents the d-c equivalent of all the noise bursts. The line, PQRS has been drawn to indicate the relative magnitudes of different parameters.

Bursts with peak amplitudes extending to the region between aa and bb like the one marked L are mostly from local sources. Bursts with peak amplitudes well below bb like the one marked $N$, are mostly from near sources. A pulse like the one indicated by $D$ is probably a peak amplitude of the background. It is clear from the figure that bursts arising from local and near sources differ only in amplitude.

The structure of a noise burst, as revealed in the record appears to be complex. Features appear to vary from burst to burst. Put in broad terms, bursts can contain large and small amplitude pulses superposed on some continuous "grass" or background. Bursts marked $A$ have large amplitude pulses only, while those marked $B$ have both large and small amplitude pulses. The time interval between the peaks of such successive pulses appear to lie in the range, 10 to $100 \mathrm{msec}$. Since this paper is concerned with noise bursts as a whole, the details of the structure of a burst will not be discussed further here.

\subsection{Noise Burst Parameters}

A noise burst in figure 1 can be idealized by a rectangular pulse $Y Y$, representing the average amplitude and duration. The parameters of interest are (a) the burst duration, (b) the time interval between successive bursts, and (c) the amplitude. All three parameters vary in a random way and have, therefore, to be dealt with in statistical terms. Even for this purpose, the three parameters have to be properly defined so that measurements of significance can be carried out. The procedure adopted for this purpose will be summarized briefly in what follows.

The audio frequency output at the detector of a superheterodyne receiver is recorded on magnetic tape and the tape played back. The interval between the time when a burst becomes audible to the time when it becomes inaudible represents the burst duration. The interval between successive bursts becoming audible represents the time interval between successive bursts. The time characteristics of noise bursts have been studied in this way quite frequently, but in routine day to day measurements, the burst rate, i.e., the number of bursts recorded per minute, is investigated.

The most desirable amplitude parameter to be measured is the average amplitude, i.e., the amplitude corresponding to $Y Y$ in figure 1 . This is difficult to measure in practice. Hence, the quasi-peak value of the amplitude is measured. This is done by feeding the output at the detector of a superheterodyne receiver to an output unit having charging and discharging time constants of 10 and 500 msec respectively, as previously described and discussed [Aiya, 1954]. The possible relationship between this quasi-peak value and the amplitude corresponding to $Y Y$ in figure 1 is at present under examination. It is needless to say that the two are related by a factor which has to be statistically evaluated. In the rest of the paper, the amplitude of a noise burst is dealt with in terms of the quasi-peak value.

\subsection{Distributions}

The assessment of noise burst parameters in statistical terms requires a study of their distributions. The conditions under which such a study should be carried out have been previously discussed in detail [Aiya, 1962]. Theoretically, there are, at any given time, many noise bursts arising from local, near, and distant sources. All of them cannot be lumped together and studied. Having regard to the distribution of the sources around a point of observation, attenuation due to propagation is not a random process. Hence, a group of noise bursts must be so selected that this difficulty is overcome.

Extensive studies have been carried out on local lightning by using the noise meter for measuring the quasi-peak value of the received noise burst field strength, recording lightning flashes with counters of different thresholds, and measuring the distances of the flashes. They indicate in quite unambiguous terms that the noise field strengths of different flashes from a limited storm area mostly lie within a range of about $20 \mathrm{~dB}$ or less. Hence, one suitable way of selecting the noise bursts to be used for studying the distribution of their parameters is to select those for which the received noise field strength is within the range stated.

In practice, we study the noise bursts of the highest amplitudes over a range of about $20 \mathrm{~dB}$. This can be done quite easily by suitably adjusting the sensitivity of the receiver. 


\subsection{Burst Rate}

If distinct and well separated noise bursts are received only occasionally, they are not of much practical significance. However, this is not the situation in tropical latitudes, where bursts are received most of the time. This is probably because the number of local and/or near sources is rather large. Further, the burst rate, i.e., the number of bursts received per minute, is quite large. This is illustrated in figure 2. This figure gives the variation of the burst rate with hour of day. One typical day has been selected for each of the four seasons for purposes of illustration. If a generalization is to be attempted, the following statement could be made. The burst rate varies between 10 and 30 per minute with an average value of the order of 20 per minute. The number of occasions when the burst rate reaches or exceeds 50 per minute is extremely rare. These figures are being furnished here in order to indicate the maximum realizable ranges of probability over which the distributions of the burst parameters can be investigated.

\section{Short Term Characteristics}

The term, 'short', is defined as a period of $5 \mathrm{~min}$ on any day during a chosen half-hour. The distributions of the three parameters of noise bursts, viz, duration, time interval between successive bursts or burst rate (which gives the average time interval), and the quasipeak noise burst field strength have been extensively investigated for periods of $5 \mathrm{~min}$ at different hours of day and on different days of the seasons. The short term characteristics thus obtained from the data collected are being described here.

A typical set of the short term 'time' characteristics as obtained is reproduced in figure 3 . The distribution is generally log-normal rather than the Poisson distribution which would be expected for random events. If rare exceptions are left out, the mean duration of a noise burst can be assumed to be 500 msec. The standard deviation is, without exception, found to be almost the same as that shown in figure 3, viz, 0.275 log-units. Ordinarily, the duration of a noise burst is of about the same value as the duration of a lightning flash as stated earlier. It does not appear to be necessary to include the study of the duration of a noise burst in continuous measurements. The time interval between successive bursts is a variable parameter and its average value can be investigated in continuous measurements by a study of the burst rate.

Typical short term 'amplitude' characteristics of noise bursts are shown in figure 4 . The distribution has been found without exception to be log-normal. The mean value is a variable parameter and has to be included for continuous investigations. It is generally found to be about $3 \mathrm{~dB}$ below the "noise burst level' which can be more easily determined in continuous measurements and has a significance which is being discussed in the next section. If exceptions

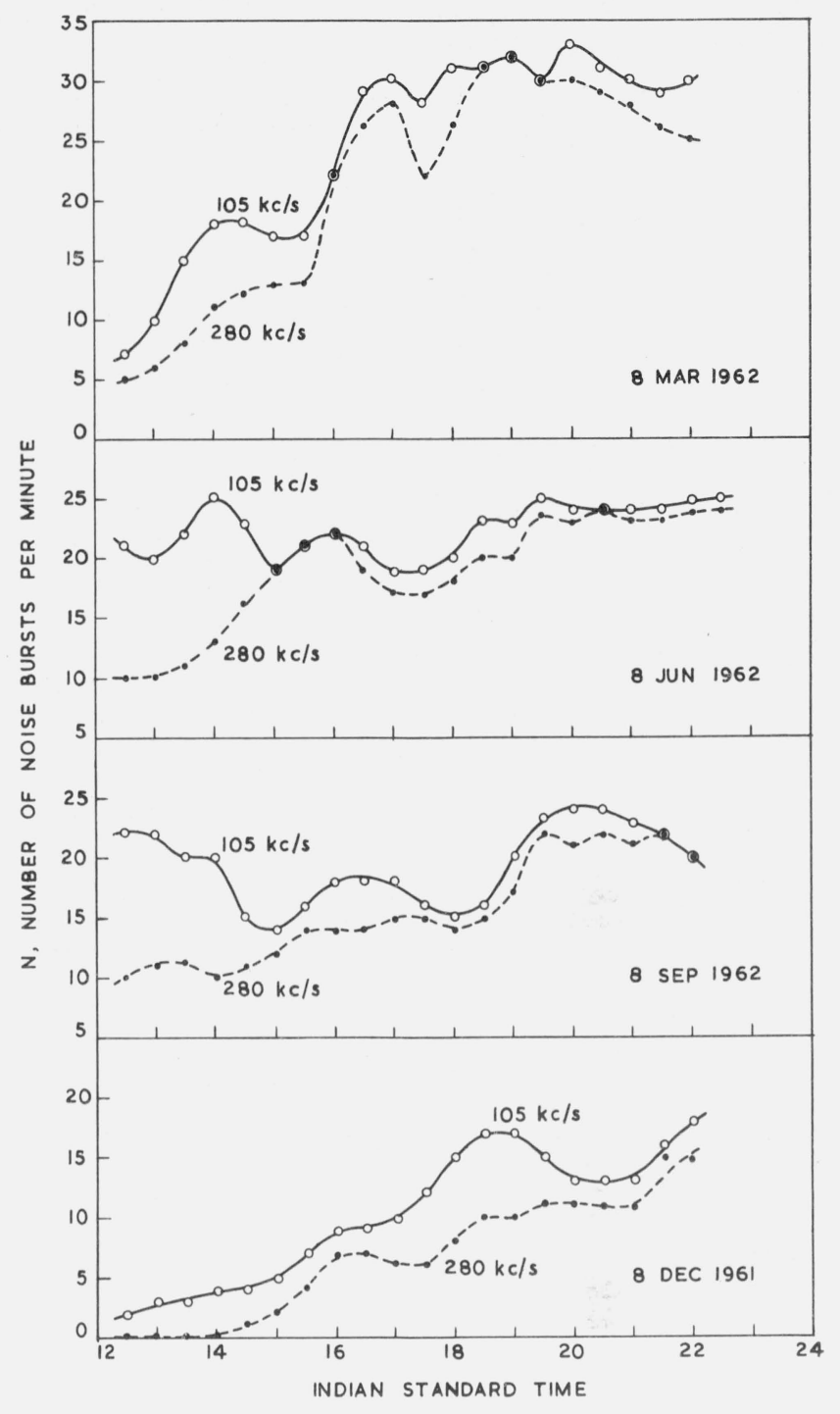

FigURE 2. Variation of noise burst rates with hour of day on typical days.

are left out, the standard deviation of the amplitude probability distribution (APD) can be assumed to lie between 3 and $4 \mathrm{~dB}$.

\section{Noise Burst Level}

Experimental results show that, on any one day, the characteristics of noise bursts remain approximately the same for a half-hour. A study of the variations of a parameter during all the days of a season at a chosen half-hour of day could be regarded as a long term characteristic. The short term mean values and standard deviations of the amplitude and the time interval between successive bursts are obviously best suited for investigations on a long term basis. Work of this type in continuous measurements is laborious. A suitable 

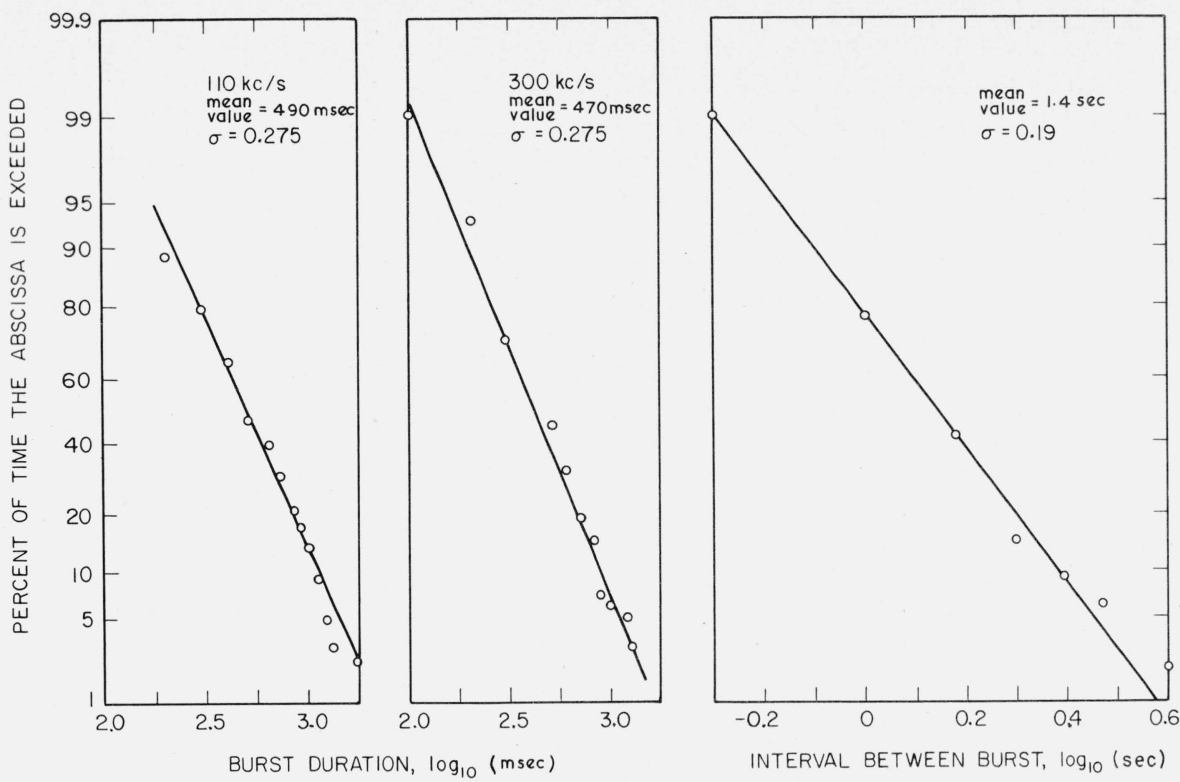

FIGURE 3. Short term 'time' characteristics of noise bursts.
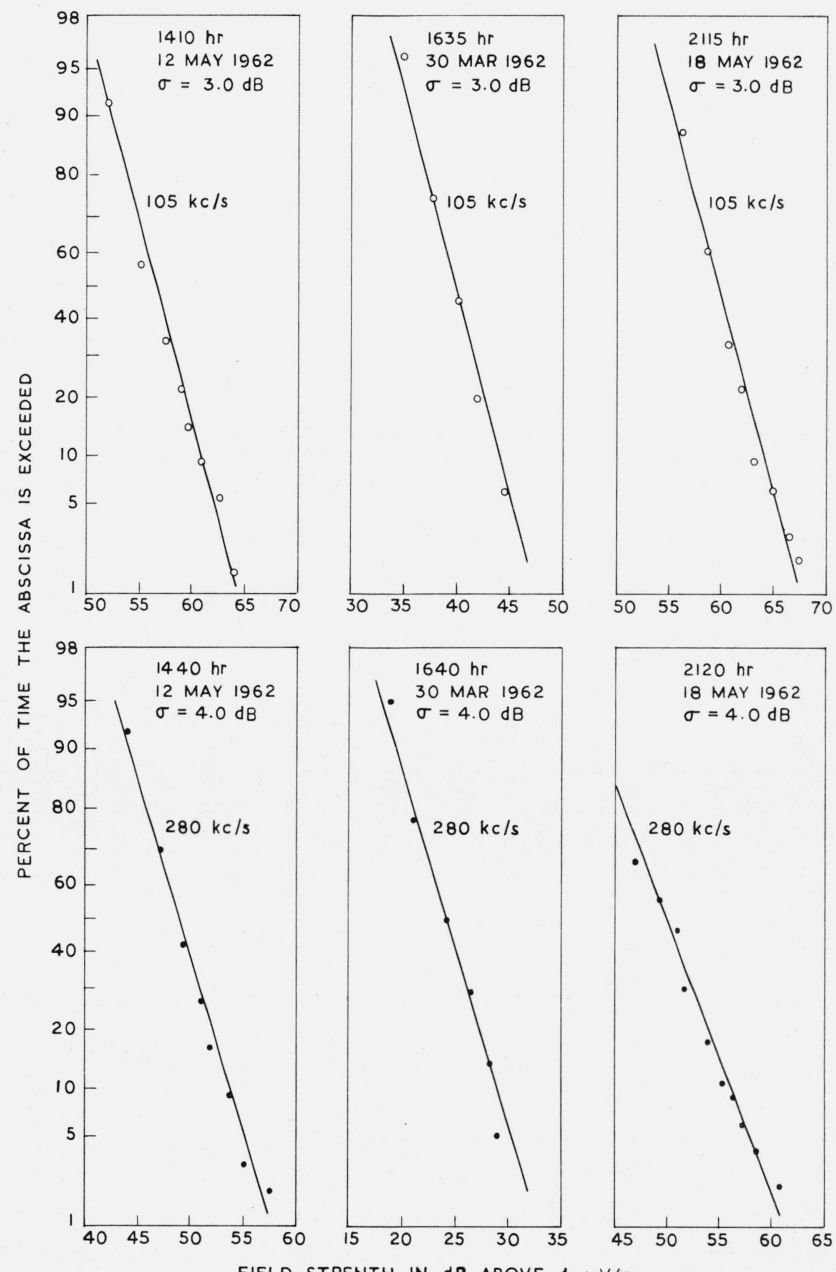

FIGURE 4. Short term 'amplitude' characteristics of noise bursts. and satisfactory alternative to this procedure was explored and this is being described and discussed here.

An examination of the data like those reproduced in figure 4 together with the fact that the mean duration of a noise burst is about $500 \mathrm{msec}$ shows that noise bursts generally occupy 10 to 25 percent of the total time. If we adopt the criterion that, for noise bursts, to be taken into account they must occupy about 10 percent of the time, we get the result that we are concerned, approximately, with the first ten highest amplitude noise bursts in a minute. If an arithmetical average of these noise bursts were taken as the noise burst level, it implies that noise burst amplitudes are below this level for 95 percent of the time. The level should therefore be an indication of the signal field strengths which are likely to suffer interference for 5 percent of the time.

The mean values of the first ten highest noise bursts per minute as calculated directly and as derived from amplitude plots such as those in figure 4 , are almost equal. But, choosing the first ten highest amplitude noise bursts per minute is equivalent to truncation of the short term APD. The effect of truncation was examined in typical cases. One set of results is reproduced in figure 5 and table 1 . They are selfexplanatory. It will also be seen that the noise burst level is $3 \mathrm{~dB}$ above the mean value of all the noise bursts recorded.

The relationship between the short term mean value of the amplitudes of all the noise bursts received and the noise burst level was examined on a long term basis. Figure 6 gives the results for a typical case. The distributions of the mean values and the noise burst levels are quite similar. The noise burst level is $3 \mathrm{~dB}$ above the mean value. This relationship can be utilized for getting mean values from the noise burst level data. 


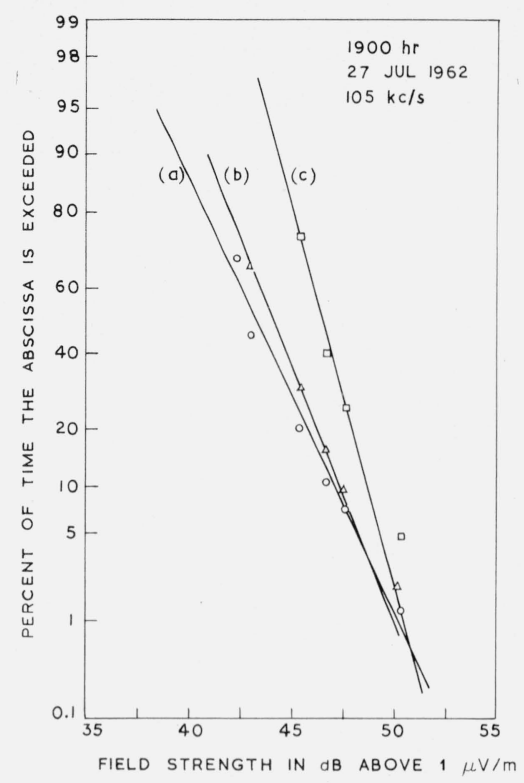

FIGURE 5. Effect of truncation on APD of noise bursts. (a) All bursts. (b) 25 highest per minute. (c) 10 highest per minute.

TABLE 1. Effect of truncation on APD of noise bursts

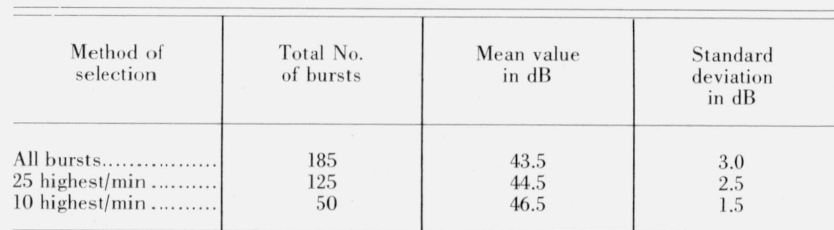

Note-Arithmetical average of the 10 highest bursts per minute was $46.9 \mathrm{~dB}$ above $1 \mu \mathrm{V} / \mathrm{m}$.

The noise burst level is thus a suitable short term parameter in terms of which the long term characteristics of noise bursts can be investigated. From this noise burst level data, the complete short term APD can be constructed as the standard deviation is known to be between 3 and $4 \mathrm{~dB}$.

Burst rate data are useful but not always quite necessary. When only the noise burst level data are available, it could be assumed that the burst rate is 20 per minute, correct to within a factor of 2 either way, and this should be adequate for most purposes.

\section{Long Term Characteristics}

Any parameter can be chosen. Its appropriate value is determined from its short term characteristics for a selected half hour of day. The distribution of these values as obtained for all the days of a season becomes a long term characteristic. Typical long term characteristics of the burst rate and its systematic variation are given in figures 7 and 8 which are selfexplanatory. (The sunset line has been drawn to correspond to the time at which the ionospheric absorption factor can be taken as zero [CRPL, 1948]. Typical long term characteristics of noise burst levels

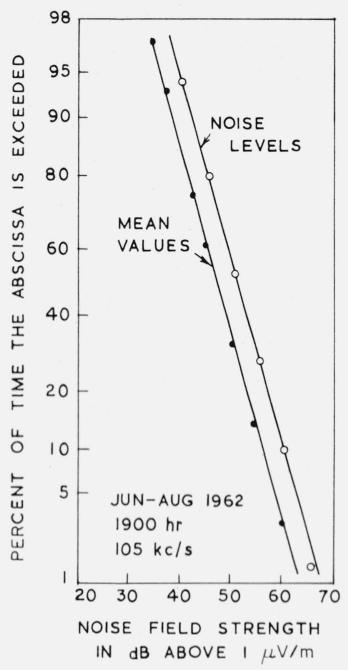

Figure 6. Seasonal APD of short term mean values of noise burst amplitudes and 'noise burst levels'.
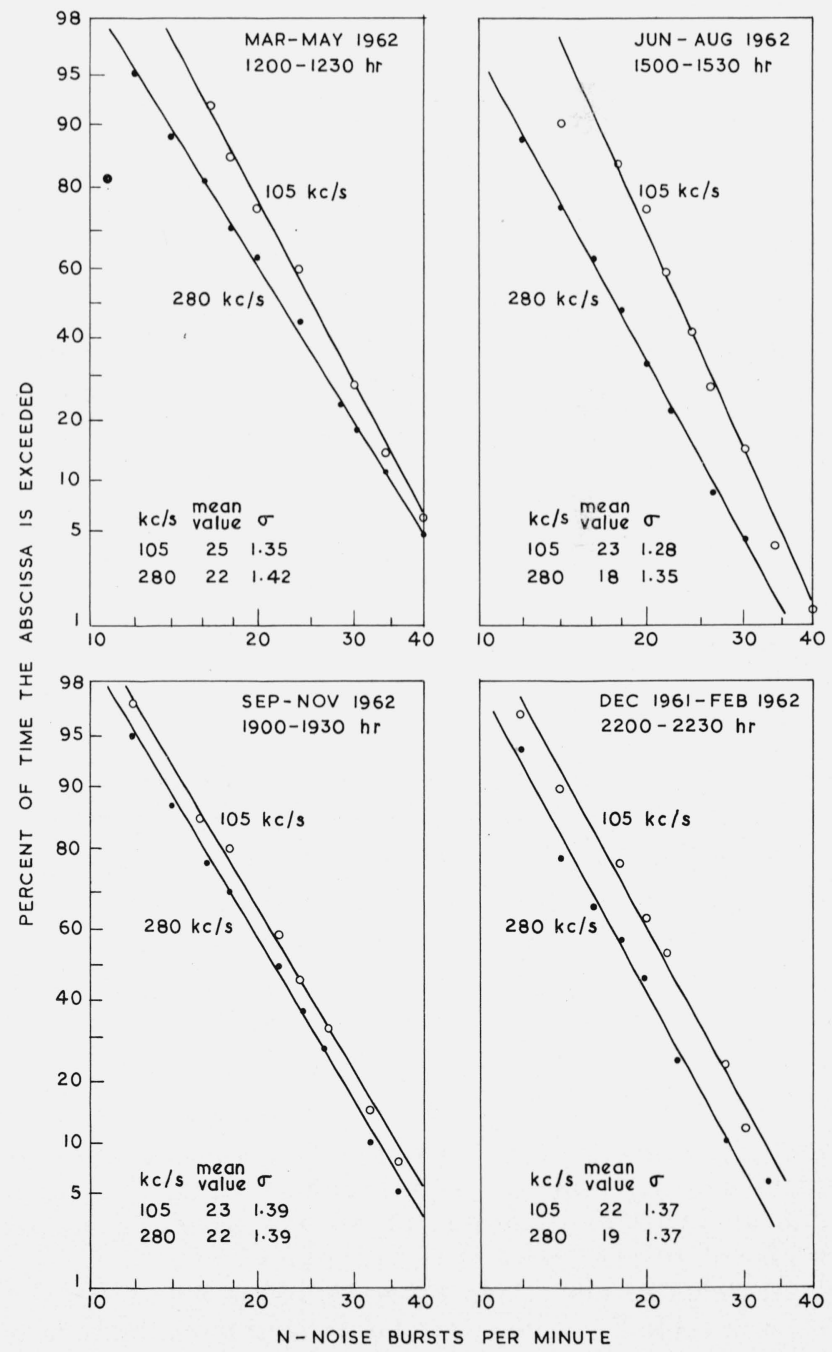

Figure 7. Typical seasonal distribution of noise burst rates. 

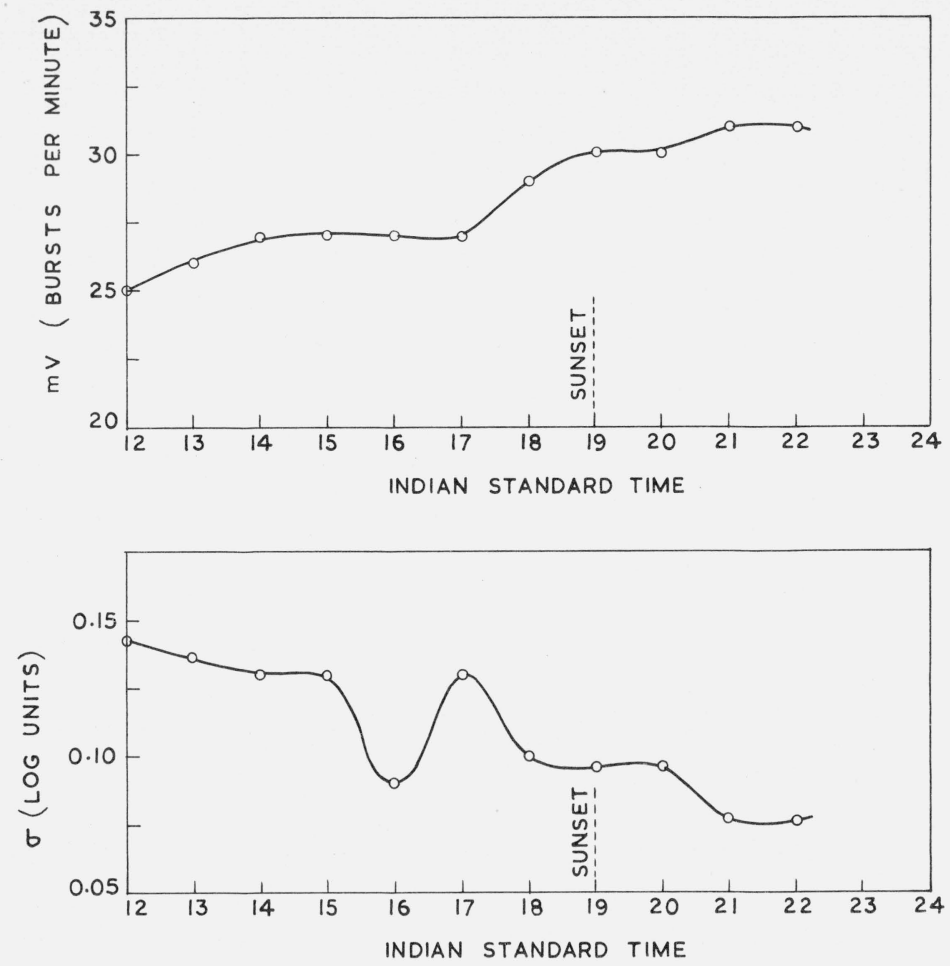

Figure 8. Mean values and standard deviations of the noise burst rates at $105 \mathrm{kc} / \mathrm{s}$ during March-May 1962.
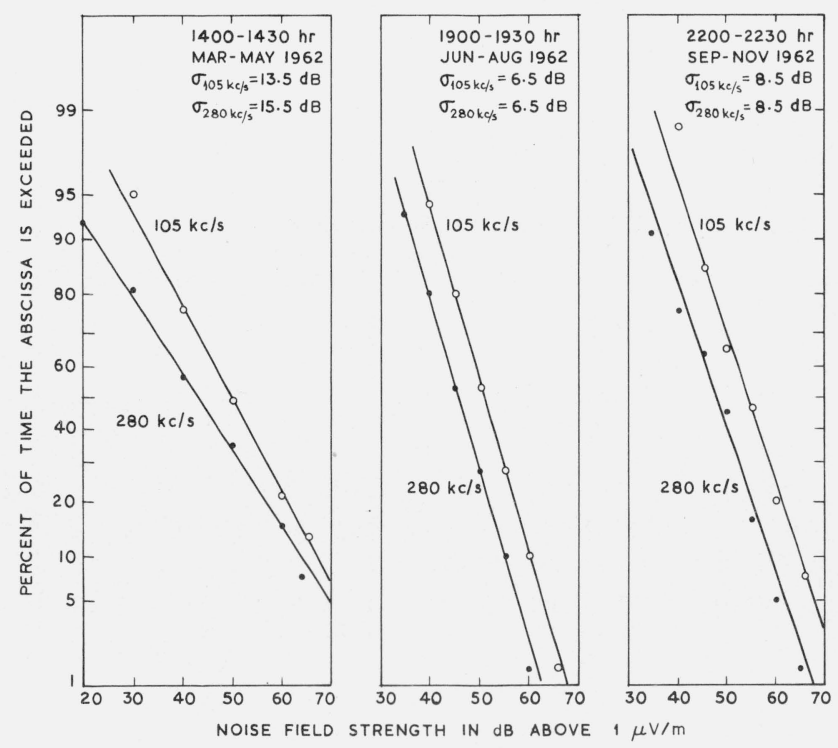

Figure 9. Typical seasonal APD of half hour 'noise burst levels'.

are given in figure 9. Their systematic variation is furnished in table 2. The tabular form has been chosen in order to bring out the frequency of occurrence of two log-normal distributions.

Systematic variations of noise burst levels can be seen in table 2. These can be utilized to examine how far the usual concept of a time block is suitable for dealing with noise bursts. For this purpose, the re-
TABLE 2. Mean values and standard deviations of noise levels during June-August 1962

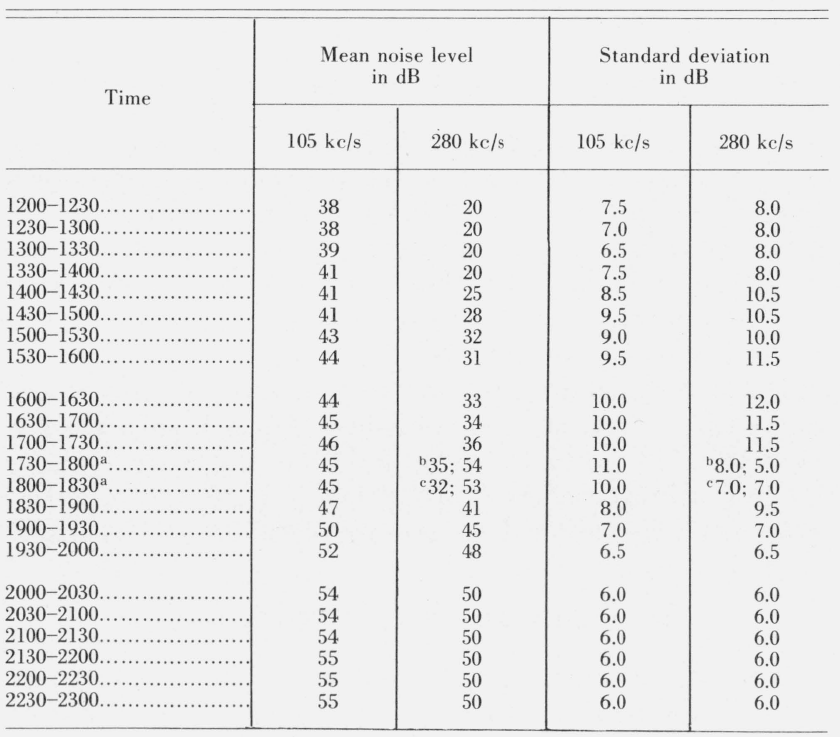

a At these half-hours it was a combination of two log-normal distributions whose parameters are given.

The second distribution contained 30 percent of the data.

" The second distribution contained 40 percent of the data.

quired information abstracted from table 2 is furnished in table 3 . It will be seen from table 3 that the mean value and the standard deviation for the noise burst level are constant for all the half-hours during 20 to 24 hr IST only. There is thus justification for treating 
TABLE 3. Averaged variation of mean values and standard deviations during June-August 1962

\begin{tabular}{|c|c|c|c|c|c|c|}
\hline \multirow{2}{*}{ Time block freq. $\mathrm{kc} / \mathrm{s}$} & \multicolumn{2}{|c|}{$1200-1600 \mathrm{hr}$} & \multicolumn{2}{|c|}{$1600-2000 \mathrm{hr}$} & \multicolumn{2}{|c|}{$2000-2400 \mathrm{hr}$} \\
\hline & 105 & 280 & 105 & 280 & 105 & 280 \\
\hline $\begin{array}{l}\text { Increase of } \mathrm{MV} / \mathrm{hr} \text { in } \\
\quad \mathrm{dB} \ldots \ldots \ldots \ldots \ldots \ldots \ldots\end{array}$ & 2 & 4 & 2 & 4 & 0 & 0 \\
\hline Standard deviation.... & $8 \pm 2$ & $10 \pm 2$ & $8 \pm 2$ & $9 \pm 3$ & 6 & 6 \\
\hline
\end{tabular}

this as a time block. During 12 to 16 and 16 to $20 \mathrm{hr}$ IST, there are systematic variations which cannot be ignored.

There is yet another long term characteristic of interest. Atmospheric noise bursts occupying about 10 percent or more of the time cannot, as a rule, be expected at a chosen half-hour on all the days of a season. Hence the percentage of days on which noise bursts occupying 10 percent or more of the time are present has to be evaluated for each half hour of day. The variation of this parameter with the hour of day becomes another long term characteristic. This is furnished in figure 10 .
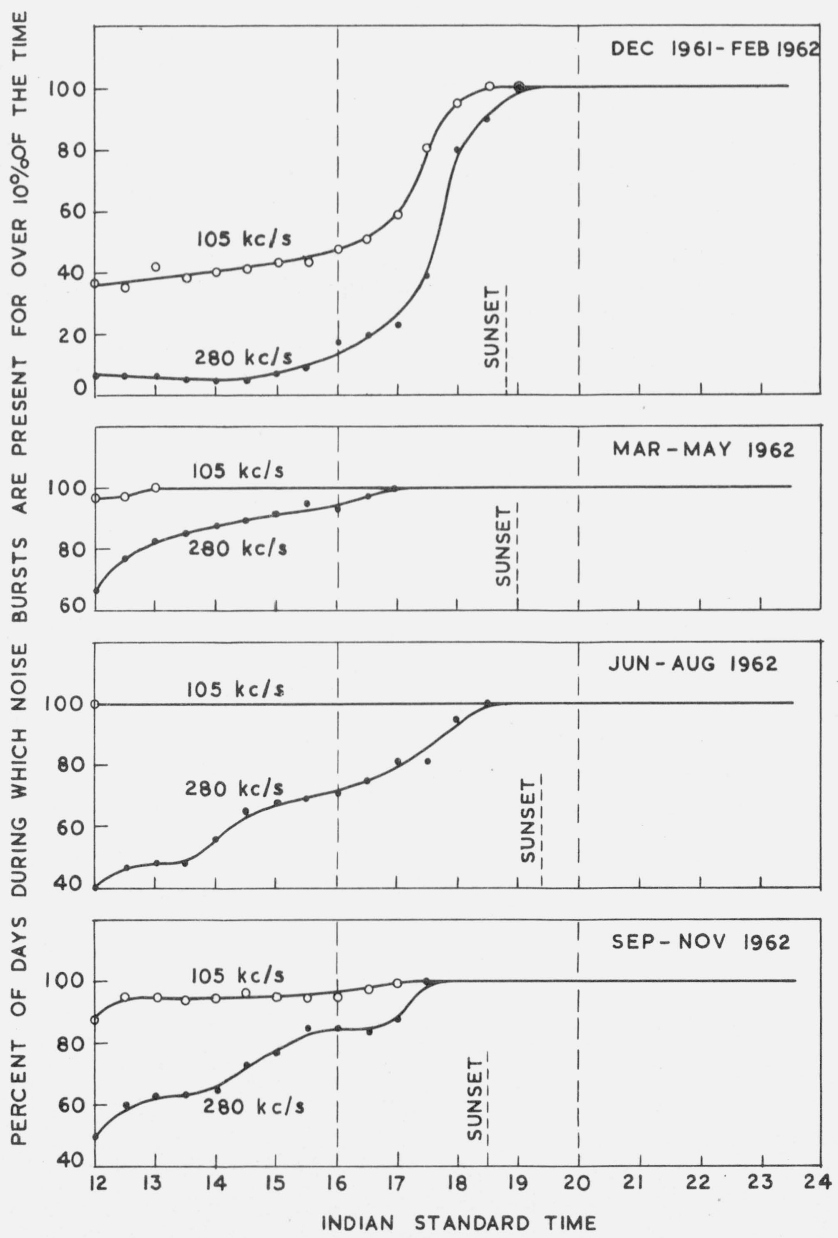

Figure 10. Seasonal variation of atmospheric noise in the form of bursts.
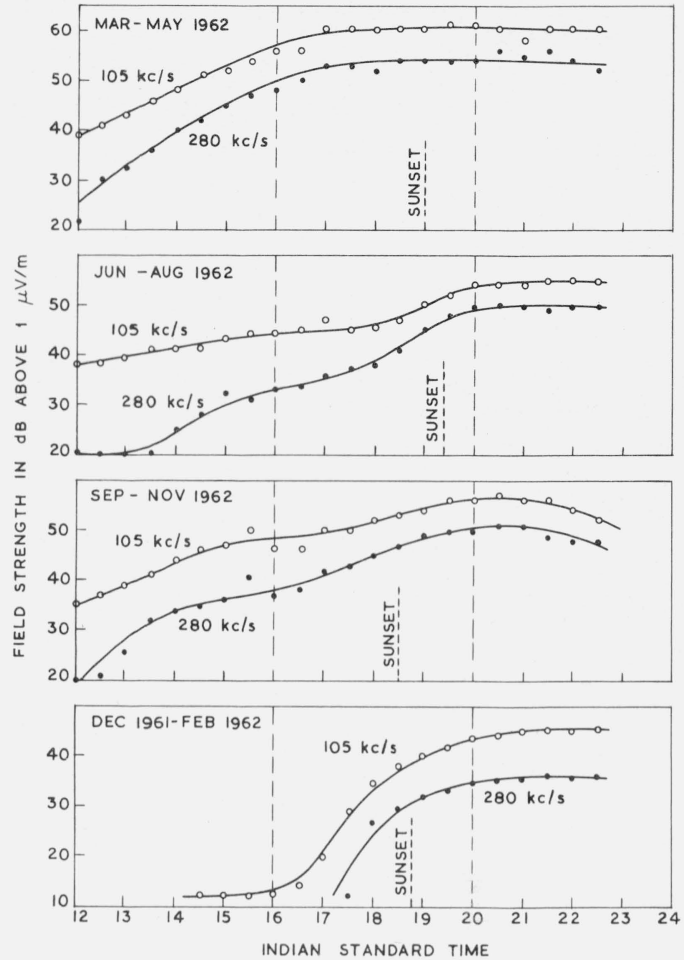

FIGURE 11. Seasonal median values of noise burst levels (quasipeak values).

\section{Design Data}

Engineers interested in voice communications may like to use the information deduced from the current investigations. In order to meet such a possible requirement, the data collected have been analyzed here, slightly differently. Systems designers think in terms of their minimum signal requirements in the presence of atmospheric noise bursts for 50 and 90 percent of the time satisfactory service. That is, they require the median and higher decile values of noise burst levels. Such values deduced from the data are given in figures 11 and 12 .

\section{Conclusion}

The characteristics of atmospheric noise bursts in the LF band have been investigated in a variety of ways over long periods of time. The investigations constitute a part of a research program on tropical thunderstorms and atmospheric radio noise. Some of the data collected for the purpose have been analyzed and presented in this paper. The number per minute and frequency of occurrence of noise bursts in tropical latitudes may be of some general interest. The short and long term distributions of the different parameters associated with noise bursts are as expected for a natural phenomenon. The long term characteristics show both systematic variations due 

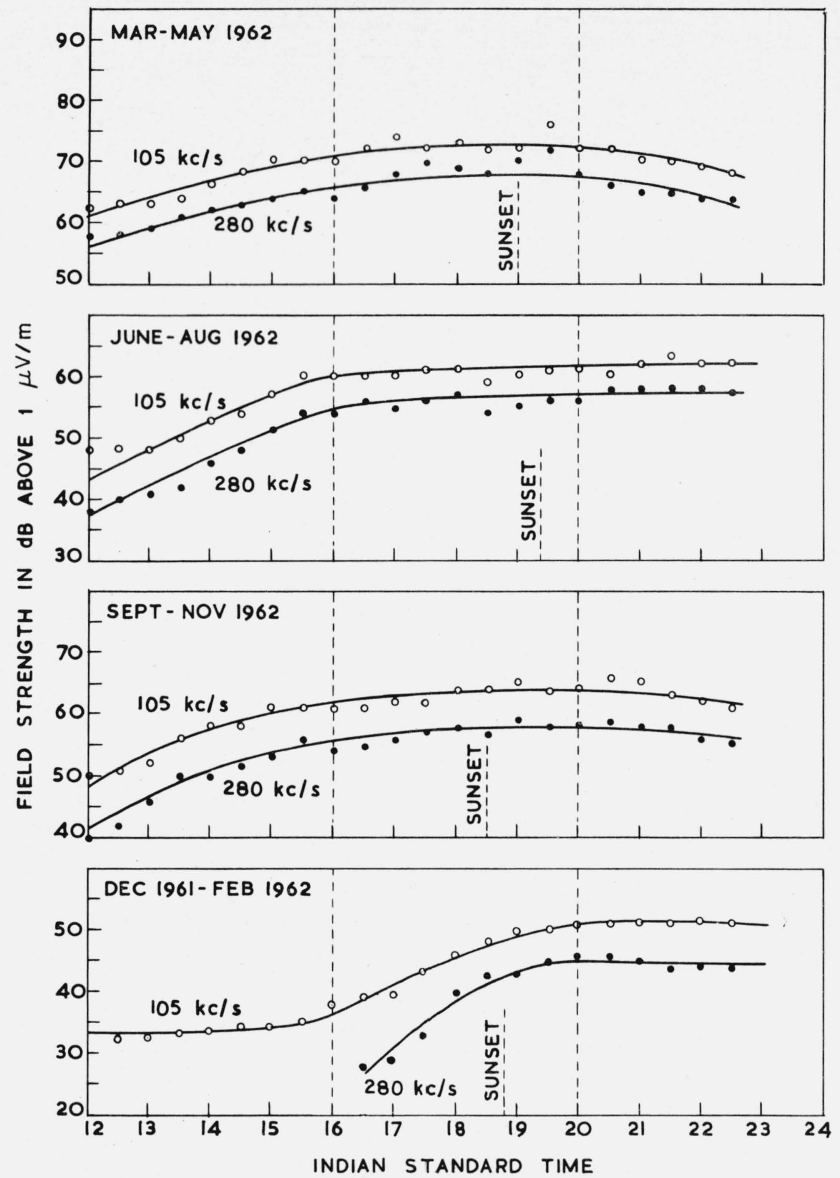

Figure 12. Seasonal higher decile values of noise burst levels (quasi-peak values).

probably to the growth and decay characteristics of local and/or near sources and changes in propagation conditions, and random variations. In Bangalore, the occurrence of distinct and well separated noise bursts in reasonably large numbers per minute appears to be more the rule rather than the exception.
Some problems, like the effect of the receiver bandwidth on noise burst parameters, the structure of noise bursts, techniques for providing noise burst data in terms similar to pulse power, etc., are still under investigation. Hence, generalized discussions have been deferred to a future communication.

Atmospheric noise data on an internationally agreed basis have been furnished by the CCIR [1964]. Since their approach to the problem differs from ours, we have not attempted a comparison of our results with their estimates.

Financial assistance received from the Council of Scientific and Industrial Research, New Delhi, is gratefully acknowledged. Some of the results reported in this paper were a part of the doctoral thesis of K. N. Lakshminarayan.

\section{References}

Aiya, S. V. C. (1954), Measurement of atmospheric noise interference to broadcasting, J. Atmospheric Terrest. Phys. 5, 230-242.

Aiya, S. V. C. (1958), Atmospheric noise interference to short-wave broadcasting, Proc. IRE 46, 580-589.

Aiya, S. V. C. (1962), Structure of atmospheric radio noise, J. Sci. Industr. Res. (India) 21D, 203-220.

CCIR (1964), World distribution and characteristics of atmospheric radio noise, Report No. 322, International Telecommunication Union, Geneva.

CRPL (1948), Ionospheric radio propagation, NBS Cir. No. 462 , US Department of Commerce, Superintendent of Documents, Washington D.C. 20402.

Lakshminarayan, K. N. (1962), Short-term 'Time' characteristics of impulsive atmospheric noise, J. Sci. Industr. Res. (India) 210 , 228-233. 